\title{
Mp10 and Mp42 from the Aphid Species Myzus persicae Trigger Plant Defenses in Nicotiana benthamiana Through Different Activities
}

\author{
Patricia A. Rodriguez, ${ }^{1,2}$ Remco Stam, ${ }^{1,2,3}$ Tim Warbroek, ${ }^{1,2}$ and Jorunn I. B. Bos ${ }^{1,2}$ \\ ${ }^{1}$ Cell and Molecular Sciences, and ${ }^{2}$ Dundee Effector Consortium, James Hutton Institute, Invergowrie, Dundee DD2 5DA, \\ U.K.; ${ }^{3}$ Division of Plant Sciences, University of Dundee, Dundee DD2 5DA, U.K.
}

Submitted 31 May 2013. Accepted 29 August 2013.

\begin{abstract}
Aphids are phloem-feeding insects that, like other plant parasites, deliver effectors inside their host to manipulate host responses. The Myzus persicae (green peach aphid) candidate effectors Mp10 and Mp42 were previously found to reduce aphid fecundity upon intracellular transient overexpression in Nicotiana benthamiana. We performed functional analyses of these proteins to investigate whether they activate defenses through similar activities. We employed a range of functional characterization experiments based on intracellular transient overexpression in $N$. benthamiana to determine the subcellular localization of Mp10 and Mp42 and investigate their role in activating plant defense signaling. Mp10 and Mp42 showed distinct subcellular localization in planta, suggesting that they target different host compartments. Also, Mp10 reduced the levels of Agrobacterium-mediated overexpression of proteins. This reduction was not due to an effect on Agrobacterium viability. Transient overexpression of Mp10 but not Mp42 activated jasmonic acid and salicylic acid signaling pathways and decreased susceptibility to the hemibiotrophic plant pathogen Phytophthora capsici. We found that two candidate effectors from the broad-host-range aphid $M$. persicae can trigger aphid defenses through different mechanisms. Importantly, we found that some (candidate) effectors such as Mp10 interfere with Agrobacterium-based overexpression assays, an important tool to study effector activity and function.
\end{abstract}

Aphids are economically important pests that cause extensive damage by feeding and transmitting important viruses to many crops worldwide. Some of the over 5,000 aphid species have a broad host range, causing damage to a variety of crops, whereas others are restricted to only a few plant species (Blackman and Eastop 2006). With genomics resources now available for various aphid species (The International Aphid Genomics Consortium 2010; Ramsey et al. 2007), there is an opportunity to identify both the plant and aphid proteins that are essential to establish interactions.

Like plant pathogens, aphids form close associations with host plants. One important step in establishing host-parasite

Corresponding author: J. I. B. Bos; E-mail: jorunn.bos@hutton.ac.uk; Telephone: +01382568700 .

* The $\boldsymbol{e}$-Xtra logo stands for "electronic extra" and indicates that four supplementary figures and one supplementary table are published online.

(C) 2014 The American Phytopathological Society associations is the delivery of effectors inside host plants to modulate host cell processes and enable colonization. In recent years, evidence has accumulated that aphids deliver effectors inside their hosts (Atamian et al. 2013; Bos et al. 2010; Hogenhout and Bos 2011; Mutti et al. 2008; Pitino and Hogenhout 2013; Rodriguez and Bos 2013; Will et al. 2007). While feeding and probing using their specialized mouthparts (or stylets), aphids deliver effector proteins mixed with saliva directly into the host-stylet interface. Since aphid saliva is being secreted into plant cells, including the phloem, and into the apoplast (Tjallingii 2006), effectors are most likely delivered into different host cell compartments. Here, these effectors are then expected to alter host cell processes by, for example, interacting with host targets to alter their mode of action and thereby promote virulence (Rodriguez and Bos 2013).

Activation and suppression of plant defenses is a common feature of plant parasite effectors. In the first layer of defense, plants can detect the presence of potential parasites by recognition of microbe-associated molecular patterns (MAMPs) to mount pattern-triggered immunity (PTI) (Jones and Dangl 2006). Successful plant pathogens, however, deliver effectors inside their hosts to suppress this and other types of plant defenses by interacting with and altering the mode-of action of important plant defense signaling components. However, plant pathogen effectors that are deployed to suppress host defenses may be recognized by plant disease resistance $(\mathrm{R})$ proteins, in particular, host genotypes, resulting in effector-triggered immunity (Jones and Dangl 2006). This recognition can occur via direct $\mathrm{R}$ protein-effector interactions or via detection of changes in the host target by an $\mathrm{R}$ protein. Although a number of $R$ genes have been identified that confer resistance to aphids in several plant-aphid interactions (Hogenhout and Bos 2011; Rodriguez and Bos 2013), the role of their encoded receptors toward recognition of the generalist aphid species Myzus persicae remains unclear. To date, several lines of evidence point to a possible role of MAMP perception in plant-aphid interactions. For example, elicitor activity has been observed in saliva of the aphid species $M$. persicae (green peach aphid) and is thought to involve a low molecular weight proteinaceous molecule (de Vos and Jander 2009). In addition, an aphid effector from $M$. persicae suppressed the reactive oxygen species (ROS) burst triggered specifically by the bacterial MAMP flg22 (Bos et al. 2010), suggesting that this effector, like a number of effectors from pathogenic microbes, plays a role in PTI suppression in plants.

With more aphid genome and transcriptome sequencing data becoming available, effector identification studies using bioinformatics- and proteomics-based approaches are increasing. 
Transcriptomics and proteomics, as well as functional genomics approaches, have revealed sets of candidate effectors from several aphid species, including Acyrthosiphon pisum (Carolan et al. 2009, 2011), Diuraphis noxia (Nicholson et al. 2012), Macrosiphum euphorbiae (Atamian et al. 2013), and Myzus persicae (Bos et al. 2010). The next step is to confirm that these candidates are, indeed, effectors as a prelude to their functional characterization. Critically, we know very little about how effectors from aphids interact with plants at the molecular level and how they impact plant cellular processes to trigger or suppress defenses or promote the release of nutrients. For a number of aphid candidate effectors, virulence activities have been identified, including Mp10 from M. persicae. Interestingly, when expressed in the host cytoplasm, Mp10 suppresses the ROS burst mediated by the bacterial MAMP flg22, pointing to a role in virulence. However, in addition, this candidate effector is able to trigger chlorosis in Nicotiana benthamiana, confer avirulence to recombinant Potato virus $X(\mathrm{PVX})$ in $N$. tabacum, and reduce aphid performance in overexpression assays (Bos et al. 2010), pointing to a role in activation of plant defense responses. Interestingly, the induction of chlorosis by Mp10 is dependent on the presence of the ubiquitin ligase SGT1, which may suggest that this protein is recognized by a nucleotide-binding site leucine-rich repeat (NBS-LRR) protein (Bos et al. 2010). These observations led to the hypothesis that Mp10 exhibits dual activities that can result in both triggering as well as suppressing plant defenses (Bos et al. 2010). Another effector candidate, Mp42, was also identified to reduce aphid performance upon overexpression inside the host cytoplasm but no potential virulence activity for this protein has been identified as yet (Bos et al. 2010). Despite the fact that the aphid species M. persicae is a generalist and able to infest plants in over 40 families, this aphid appears to secrete proteins, such as Mp10 and Mp42, that activate plant defenses when expressed inside the host cytoplasm. The molecular mechanisms involved in manipulation of host defenses by Mp10 and Mp42 and the role of these candidate effectors in plant-aphid interactions remain elusive.

We performed functional analyses to investigate whether the aphid candidate effectors Mp10 and Mp42 reduce aphid performance in a similar manner, and to gain insight into the molecular basis of their activities. Subcellular localization studies of green fluorescent protein (GFP)-Mp10 and GFP-Mp42 fusion proteins showed that these candidate effectors localize to different cellular compartments. In addition, we found that Mp10 but not Mp42 interfered with Agrobacterium-mediated protein overexpression in planta. Using quantitative polymerase chain reaction (PCR), we showed that the effect of Mp10 on Agrobacterium-mediated protein expression corresponded with a reduction in detectable transcript levels. In addition, we assessed gene expression levels of several markers of the jasmonic acid (JA)-, and salicylic acid (SA)-dependent defense signaling pathways upon Mp10 and Mp42 overexpression. Surprisingly, all markers showed a significant induction in the presence of Mp10 but not Mp42, indicating that transient overexpression of Mp10 activates defenses via several pathways and alters the plant hormone balance. Despite this, we did not observe any effects of Mp10 on Agrobacterium viability. However, overexpression of Mp10 but not Mp42 decreased virulence of the oomycete plant pathogen Phytophthora capsici. Altogether, these data suggest that Agrobacterium-mediated overexpression of Mp10 alters plant hormone signaling and reduces host susceptibility to aphids and a plant pathogen. And, although Mp10 and Mp42 both reduce aphid performance when overexpressed in the host cytoplasm, they exhibit different activities, which can be exploited to study the processes associated with disease and immune signaling in plants.

\section{RESULTS}

Aphid candidate effectors Mp10 and Mp42

localize to different subcellular compartments in host cells.

The subcellular localization of effectors can provide information about the potential action site of these proteins inside the plant cell. We determined and compared the localization patterns of two aphid candidate effectors, Mp10 and Mp42, which both reduce aphid fecundity when overexpressed inside the cytoplasm of $N$. benthamiana (Bos et al. 2010). For this purpose, we transiently overexpressed the GFP-Mp10 and GFP-Mp42 fusion proteins in $N$. benthamiana plants using Agrobacterium. Two days postinfiltration (dpi), fluorescence of GFP-Mp10 was detected by confocal microscopy in the host cytoplasm (Fig. 1A), whereas fluorescence of GFPMp42 appeared to be mainly associated with membranes and aggregation products, as well as smaller aggregates, or puncta, surrounding the nuclear envelope (Fig. 1A). Using confocal microscopy, we observed that the larger aggregation products containing GFP-Mp42 were motile within the host cytosol (data not shown). Although both GFP-Mp10 and GFP-Mp42 are small enough proteins to passively diffuse into the nucleus, we observed a GFP signal for Mp10 inside the nucleus similar to the GFP control but not for Mp42 (Fig. 1A). To confirm that full-length GFP-fusion proteins were expressed, we performed Western blot analysis (Supplementary Fig. S1). This showed that both proteins were successfully expressed in planta, with GFP-Mp42 detectable at slightly higher levels than GFP-Mp10. We detected full-length versions of GFP-Mp10 ( $\pm 44 \mathrm{kDa})$ and GFP-Mp42 $( \pm 48 \mathrm{kDa})$, as well as one $\pm 35-\mathrm{kDa}$ degradation product of GFP-Mp10 and two degradation products $( \pm 37$ and $\pm 27 \mathrm{kDa}$ ) of GFP-Mp42. Due to the overall low GFP-fusion protein detection levels, we performed protein purification prior to Western blotting, which could have resulted in the observed degradation. It is also possible that these proteins are processed in the host cytosol as part of a relevant biological process resulting in shorter peptide fragments.

In order to investigate the localization of GFP-Mp42 in more detail, we transiently expressed GFP-Mp42 and labeled the cells with the endocytic membrane marker FM4-64, which binds to plasma membrane lipids and follows the endocytic internalization pathways (Vida and Emr 1995). GFP-Mp42 colocalized with this marker along the plasma membrane, and some of the GFP-Mp42 aggregation products were observed at the plasma membrane as well (Fig. 1B). We also transiently coexpressed GFP-Mp42 with mRFP-HDEL, a known endoplasmic reticulum (ER) retention signal (Denecke et al. 1992) fused to mRFP, to determine whether Mp42 colocalized with the ER. Interestingly, both the GFP-Mp42 and HDEL fluorescent signals were detected in the motile aggregation products throughout the cell, in line with the ER being a dynamic organelle. However, the GFP-Mp42 puncta that were observed around the nuclear envelope only partly colocalized with the ER marker (Fig. 1B). This suggests that, unlike GFP-Mp10, GFP-Mp42 localizes to the plasma membrane and partly colocalizes in motile ER aggregation products throughout the cell.

To determine whether Mp42 triggers the formation of motile aggregation products that colocalize with the ER, we expressed the mRFP-HDEL marker together with GFP (control), GFPMp42, or untagged Mp42. This showed that the motile aggregates were formed specifically in the presence of both GFP-Mp42 and untagged Mp42 but not in the presence of GFP alone (Fig. 1C and D). Therefore, Mp42 may exhibit an activity that causes the formation of motile ER aggregation products. 


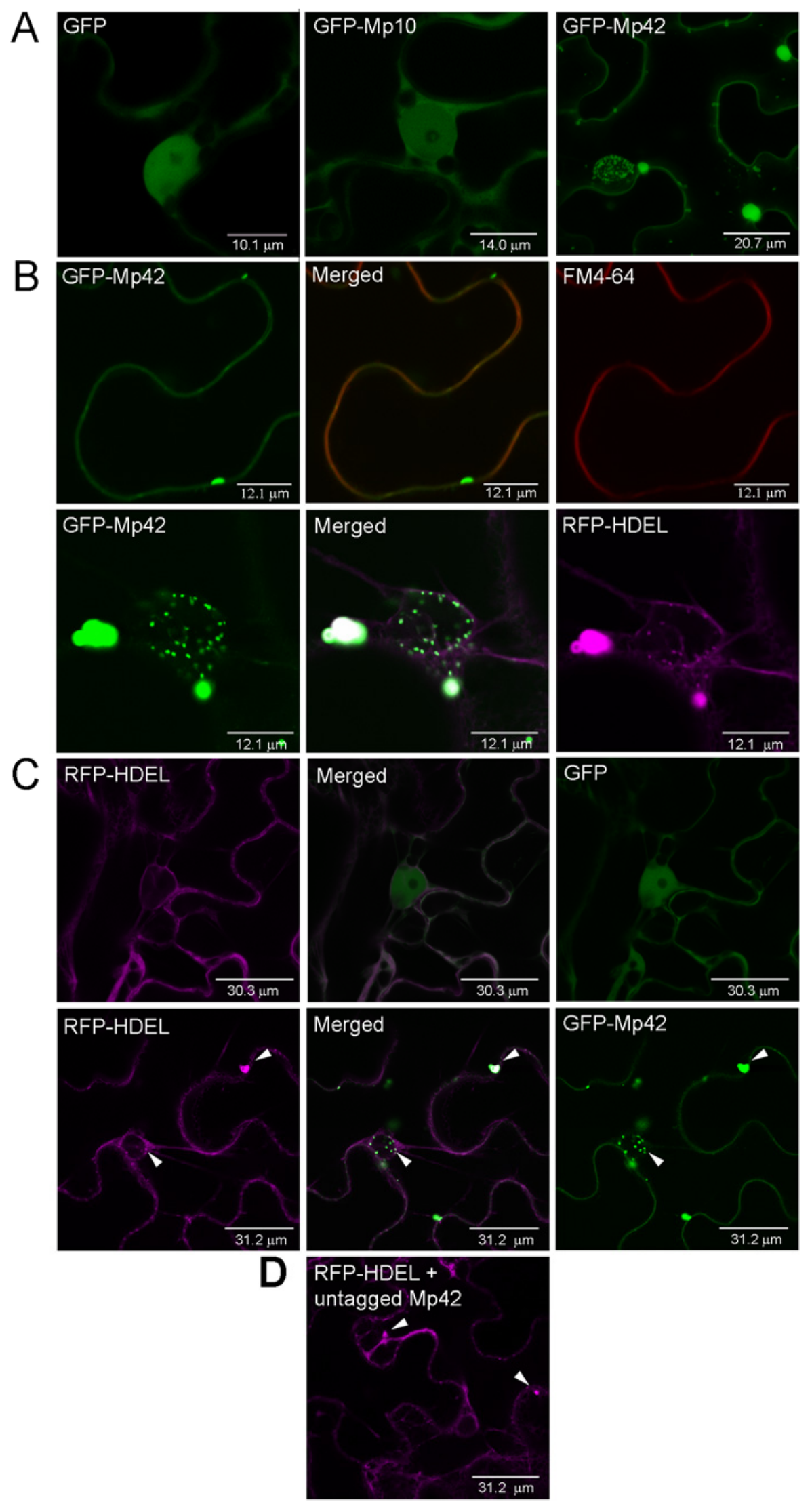

Fig. 1. Subcellular localization of green fluorescent protein (GFP)-Mp10 and GFP-Mp42 fusion proteins. GFP- and red fluorescent protein (RFP)-fusion proteins were transiently expressed in Nicotiana benthamiana using agroinfiltration and localized $48 \mathrm{~h}$ after infiltration. A, Confocal laser-scanning microscopy of GFP (control), GFP-Mp10, and GFP-Mp42. B, Confocal laser-scanning microscopy of GFP-Mp42 in the presence of FM4-64 and coexpressed with the endoplasmic reticulum (ER) retention signal mRFP-HDEL. C, Confocal laser-scanning microscopy of GFP (control) and GFP-Mp42, coexpressed with the ER retention signal mRFP-HDEL. D, Confocal laser-scanning microscopy of the ER retention signal mRFP-HDEL in the presence of untagged Mp42. White bars represent the individual scales annotated in each picture and white arrows point to Mp42-mediated aggregation products. 
Mp10 but not Mp42 affects

Agrobacterium-mediated protein expression.

While performing coexpression assays of several aphid candidate effectors with tagged fusion proteins in planta, we discovered that Mp10 reduced detectable protein levels of other (plant) proteins regardless of their tags. To investigate this in detail, we performed a time-course experiment where we coexpressed GFP together with Mp10 (both FLAG-tagged and untagged) and harvested agroinfiltrated leaf materials 2 to 4 dpi. This showed that both untagged (Fig. 2A) and FLAGtagged Mp10 (Supplementary Fig. S2A) reduced steady-state levels of GFP at all time points tested. Moreover, detectable Mp10 levels were reduced over time, suggesting that Mp10 also negatively impacts its own Agrobacterium-mediated expression. Furthermore, we showed that Mp10 affects not only the expression of GFP but also of GFP-fusion plant proteins. For example, coexpression of FLAG-Mp10 with GFP-StCSN5, a Solanum tuberosum protein with similarity to Arabidopsis CSN5 (COP9 signalosome complex subunit 5), reduced detectable levels of StCSN5. To rule out that Mp10 specifically reduces the levels of GFP or GFP-fusion proteins, we also coexpressed GFP-Mp10 with FLAG-StCSN5, which resulted in reduced detection levels of FLAG-StCSN5. Co-expression assays of StCSN5 and Mp10 where performed in the presence of $\mathrm{p} 19$, which indicates that the reduction in protein levels is not due to silencing over time.

We also investigated whether Mp10 affected protein levels of GFP expressed in $N$. benthamiana transgenic lines by coexpression with FLAG-Mp10 over a 2- to 4-day time course.

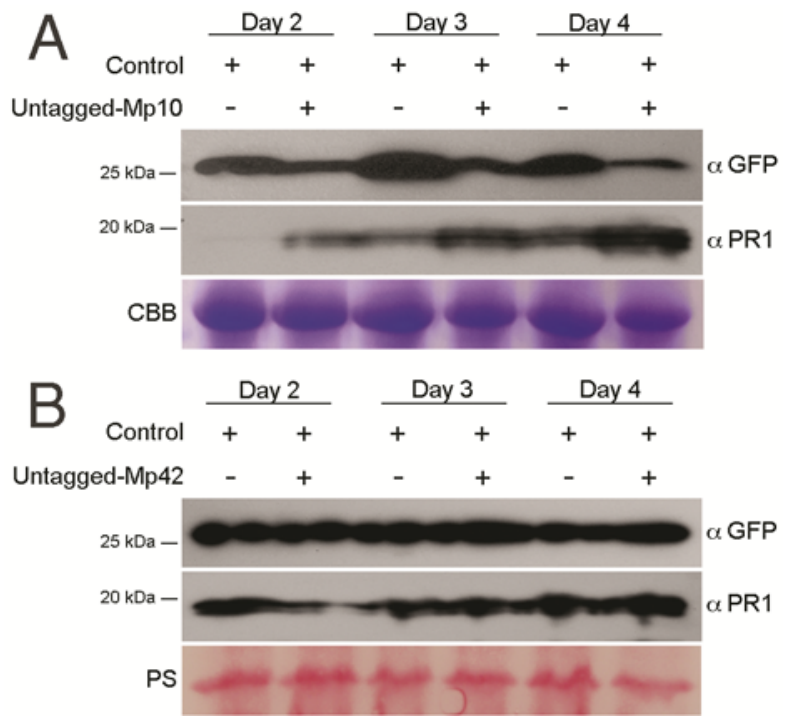

Fig. 2. Mp10 reduces detectable levels of Agrobacterium-mediated protein expression. A, Expression levels of transiently overexpressed green fluorescent protein (GFP) and endogenous pathogenesis-related 1 (PR-1) proteins in the presence of Mp10. Western blot analysis was performed on leaf samples from Nicotiana benthamiana leaves transiently co-expressing GFP with Mp10 (+) or the vector control (-). Protein levels were analyzed during a time-course experiment from 2 to 4 days postinfiltration. Proteins were analyzed by immunoblotting using GFP- and PR1-specific antibodies. Coomassie blue staining $(\mathrm{CBB})$ was used to show equal loading. B, Expression of GFP and PR-1 proteins in the presence of Mp42. Western blot analysis was performed on samples from $N$. benthamiana leaves transiently co-expressing GFP with Mp42 (+) or the vector control (-). Protein levels were analyzed during a time-course experiment from 2 to 4 days postinfiltration. Ponceau S staining (PS) was used to show equal loading. Proteins were analyzed by immunoblotting using GFP- and PR1-specific antibodies. Experiments were repeated at least two times with similar results.
Western blot analyses of the time-course materials showed that Mp10 did not affect detectable GFP levels in transgenic lines, indicating that Mp10 specifically reduces Agrobacteriummediated expression (Supplementary Fig. S3).

To test whether the effect of Mp10 on Agrobacterium-mediated overexpression of proteins is related to increased proteasomal degradation, we infiltrated the $26 \mathrm{~S}$ proteasome inhibitor epoxomicin $16 \mathrm{~h}$ before harvesting leaf tissue coexpressing Mp10 and GFP for Western blot analysis. However, epoxomicin did not affect the Mp10-mediated reduction of protein overexpression by Agrobacterium (Supplementary Fig. S4), suggesting that this activity of Mp10 is not dependent on the $26 \mathrm{~S}$ proteasome.

We compared Mp10 and Mp42 in similar time-course coexpression experiments to determine whether Mp42 also affects Agrobacterium-mediated expression of proteins or whether this activity is specific to Mp10. Here, we opted to use untagged Mp42 to prevent any possible interference of tags with activity. This also allowed us to compare any differences in putative effector activity with the observation that $35 \mathrm{~S}$-based overexpression of untagged Mp10 and Mp42 reduced aphid fecundity to similar levels. In contrast to Mp10, we found that Mp42 does not affect the detectable protein levels of GFP (Fig. 2B), suggesting that these two aphid effector proteins exhibit different activities in planta.

\section{Mp10 affects Agrobacterium-mediated protein expression at the transcriptional level.}

Our results suggest that reduced steady-state levels of coexpressed proteins are not due to degradation, indicating that Mp10 acts on the transcriptional level. To investigate whether Mp10 reduced Agrobacterium-mediated expression at the transcriptional rather than post-translational level, we performed a time-course experiment where we analyzed the transcript accumulation of GFP by real-time PCR from 2 to 4 dpi upon coexpression with Mp10 or a vector control. We found that coexpression with Mp10 decreased the level of GFP transcript accumulation from 2 dpi onward as compared with the vector control (Fig. 3). This reduction in transcript accumulation corresponds to the observed reduction in detectable protein levels of GFP upon coexpression with Mp10 by Agrobacterium.

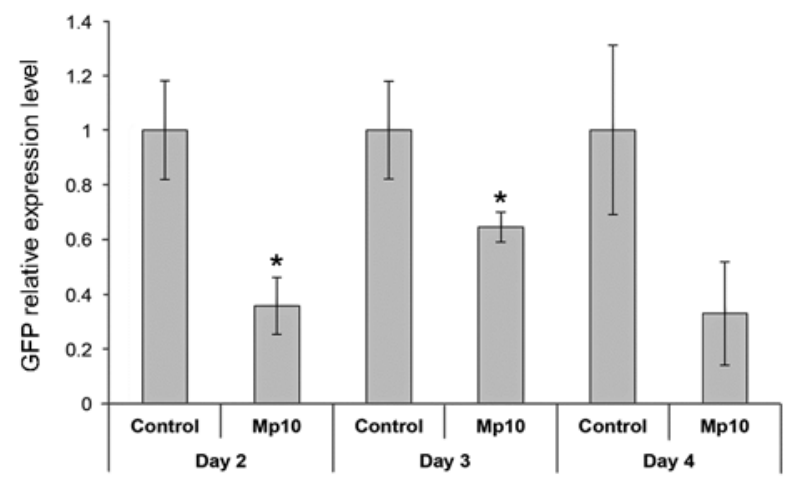

Fig. 3. Green fluorescent protein $(G F P)$ transcript abundance is decreased upon transient co-expression with Mp10 by agroinfiltration in Nicotiana benthamiana. Expression of transiently overexpressed GFP during a timecourse experiment from 2 to 4 days postinfiltration measured by quantitative real-time polymerase chain reaction. The GFP construct was coexpressed with FLAG-Mp10 (Mp10) or the vector control (control) by agroinfiltration. Transcript abundances of Mp10 samples are shown relative to the vector control samples and values were normalized to endogenous actin expression. Means and error bars representing \pm standard errors are from three replicates. Asterisks $(*)$ indicate significant differences of treatments compared with the vector control at each time point (paired $t$ test, $P<0.05$ ). Experiment was repeated two times with similar results. 
Mp10 increases expression of marker genes for hormone-related defense responses.

Both the Mp10 and Mp42 effectors are thought to trigger unknown defenses active against aphids, resulting in reduced aphid fecundity (Bos et al. 2010). In addition, we have shown here that Mp10 interferes with Agrobacterium-mediated expression, potentially by interfering with virulence mechanisms. To gain further insight into the possible role of Mp10 in triggering plant defense responses, we tested whether this candidate effector triggers marker genes of JA- and SA-dependent signaling pathways.

First, we used an antibody specific to the pathogenesisrelated 1 (PR-1) protein to detect and compare the levels of $N$. benthamiana PR-1 protein in the presence and absence of Mp10 upon agroinfiltration. Western blot analysis showed that there was an increased level of detectable PR-1 protein in the presence of Mp10 from 2 to 4 dpi (Fig. 2A). To determine whether the induction of PR-1 expression was specific to Mp10, we also analyzed PR-1 levels in the presence of Mp42 upon agroinfiltration in $N$. benthamiana. However, unlike Mp10, Mp42 did not alter the levels of detectable PR1 protein (Fig. 2B).

To gain further insight into the induction of defense-related marker genes, we performed real-time PCR experiments with gene-specific primers for SA-dependent marker genes $\mathrm{NbPRI}$ (Yoon et al. 2009) and SlGluA (Milling et al. 2011), and JAdependent marker genes NbPR4 (Maimbo et al. 2010) and
NbTPl (Yoon et al. 2009). Surprisingly, we found that overexpression of Mp10 resulted in upregulation of marker genes for both the SA-dependent (Fig. 4A) as well as the JA-dependent signaling pathways from 2 to 4 dpi. More specifically, NbPRI and SlGluA were upregulated to approximately 25 -fold and 14fold, respectively, in the presence of Mp10. In addition, $N b P R 4$ and $N b T P 1$ were upregulated up to approximately 50fold and 5-fold, respectively, in the presence of Mp10. Overall, the strongest induction of marker gene expression was observed at 2 and 3 dpi and the induction of expression was reduced at 4 dpi (Fig. 4A and B). In contrast, no induction of marker genes was observed upon transient overexpression of Mp42 (Fig. 4B). However, a slight but, in some cases, significant reduction in expression of some of the marker genes was observed in the presence of Mp42 at later time points.

Because $P A D 4$ is required for defense against $M$. persicae (Pegadaraju et al. 2005) in Arabidopsis and is known to regulate the SA-dependent signaling pathway (Feys et al. 2001; Jirage et al. 1999; Zhou et al. 1998), we set out to investigate whether Mp10 affects the expression of this gene in $N$. benthamiana. Using real-time PCR with $N b P A D 4$-specific primers, we found that NbPAD4 was upregulated to approximately sixfold upon transient overexpression of Mp10 (Fig. 4C), pointing to a role of $N b$ PAD4 in the plant defense responses triggered by Mp10. No induction of NbPAD4 was detected upon transient overexpression of Mp42, again showing that this activity is specific to Mp10 (Fig. 4C).
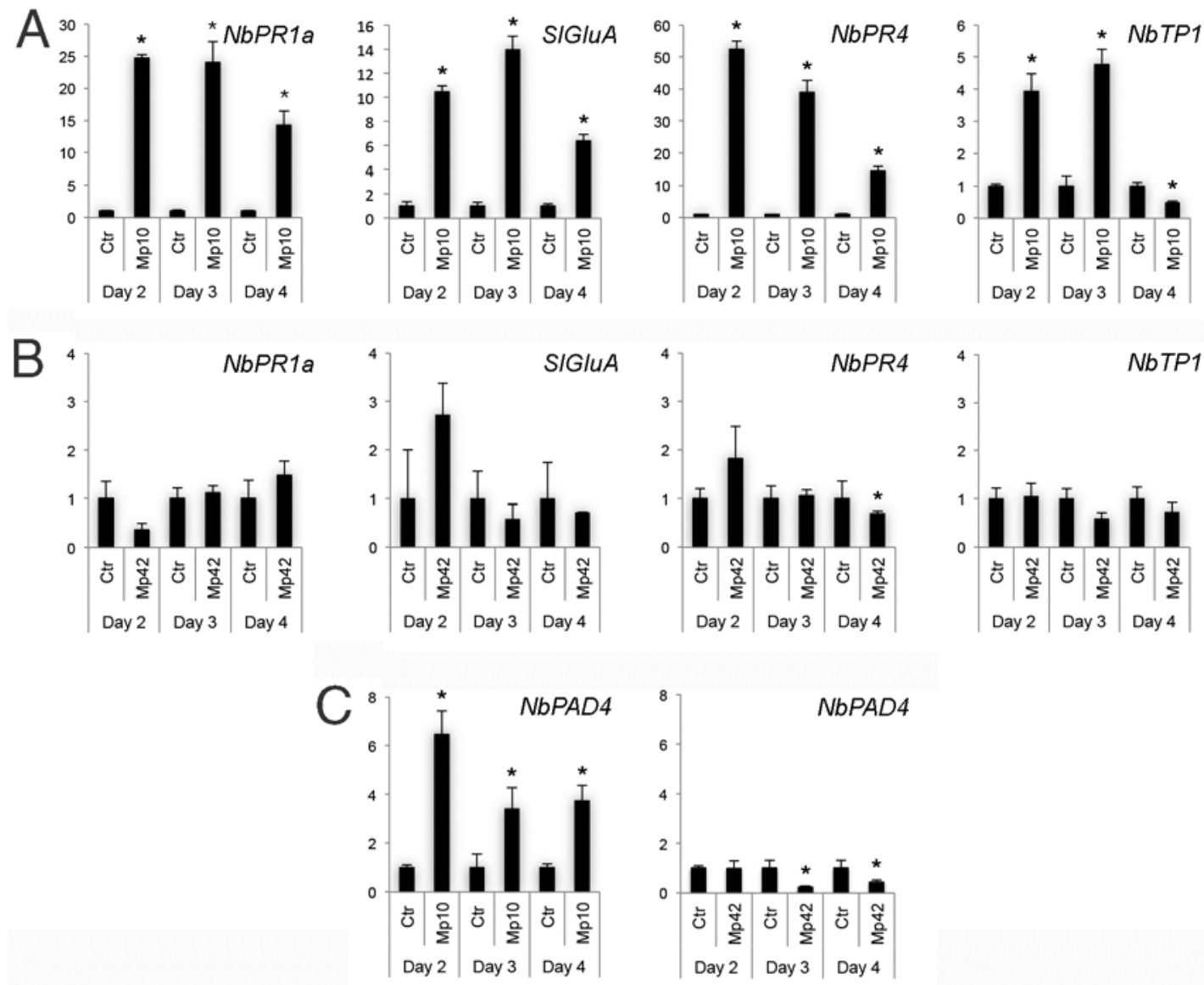

Fig. 4. Expression of marker genes for hormone-related defense responses upon transient overexpression of Mp10 and Mp42 in Nicotiana benthamiana. A and B, Expression of salicylic acid (SA)-related marker genes ( $N b P R 1$ and SlGluA) and of jasmonic acid (JA)-related marker genes (NbPR4 and NbTP1) during a time-course experiment from 2 to 4 days postinfiltration measured by quantitative real-time polymerase chain reaction upon transient overexpression of Mp10, Mp42, or the corresponding vector control (Ctr). C, Expression of NbPAD4 upon transient overexpression of Mp10, Mp42, or the corresponding Ctr. Transcript abundances of the Mp10 and Mp42 samples are shown relative to the Ctr samples and values were normalized to endogenous actin expression. Means and error bars representing \pm standard error are from three replicates. Asterisks (*) indicate significant differences of treatments compared the $\mathrm{Ctr}$ at each time point (paired $t$ test, $P<0.05$ ). 


\section{Mp10 does not affect Agrobacterium viability and triggers defense responses that reduce host susceptibility to $P$. capsici.}

The finding that Mp10 reduced Agrobacterium-mediated expression at the transcript level and triggered a range of plant defense responses suggests that this protein may affect Agrobacterium viability in planta. To test this, we overexpressed FLAG-Mp10 or the vector control by agroinfiltration in $N$. benthamiana together with an Agrobacterium strain carrying the pB7WGF2 vector to express GFP. Leaf tissues were harvested at 0 and $3 \mathrm{dpi}$ and processed for dilution plating on spectinomycin plates to select for Agrobacterium strains carrying the pB7WGF2 vector. No differences were observed between the number of colony forming units (CFU) at day 0 or day 3 in the presence or absence of Mp10, indicating that Mp10 does not affect viability of Agrobacterium cells in planta (Fig. $5 \mathrm{~A})$.

The activation of SA- and JA-signaling pathways by Mp10 suggests that this effector activates plant defenses active against pathogens and pests. To investigate whether aphid candidate effectors Mp10 and Mp42 have the potential to affect host susceptibility to a hemibiotrophic plant pathogen, we performed infection assays with the oomycete $P$. capsici on $N$. benthamiana leaves transiently expressing Mp10, Mp42, or a vector control. Lesion size diameter was measured 2 days after inoculation with $P$. capsici. We found that lesion diameters were significantly smaller in Mp10-expressing leaf tissue compared with the Mp42- or vector control-expressing leaf tissue, indicating that $P$. capsici infection was delayed by Mp10 (Fig. 5B). However, despite the delay, lesion formation and sporulation were visible in leaf tissue overexpressing Mp10 (data not shown). Mp10 was able to delay but not stop the $P$. capsici infection process and, thus, activates defenses effective against a hemibiotrophic plant pathogen.

\section{DISCUSSION}

The effector paradigm in plant-aphid interactions has only recently become evident. With the availability of aphid genomics resources as well as proteomics tools to identify secreted candidate effectors in aphid saliva, the next step is to investigate the role of these proteins in plant-aphid interactions and characterize their functions. Already, several aphid salivary proteins have been identified that share functional features with effectors of plant pathogens (Atamian et al. 2013; Bos et al. 2010; Pitino and Hogenhout 2013). These include $M$. persicae Mp10 and Mp42, which were both found to reduce aphid performance when transiently overexpressed in the cytoplasm of $N$. benthamiana, suggesting that these proteins may trigger plant defense responses. Here, we report on further characterization of these candidate effectors to determine if their activities are based on shared underlying molecular mechanisms. We found that Mp10 and Mp42 showed different subcellular localization patterns when expressed as GFP-fusion proteins in N. benthamiana. Further evidence for different activities was provided by the finding that Mp10 but not Mp42, when transiently overexpressed by Agrobacterium, activates multiple defense pathways and Agrobacterium-based expression, as well as reduces host susceptibility to the oomycete plant pathogen $P$. capsici.

The different subcellular localizations of GFP-Mp10 and GFP-Mp42 suggest that these proteins have distinct sites of activity. Subcellular localization studies of plant pathogen effectors have revealed that they target different cellular compartments in plants upon overexpression and, for some effectors, this subcellular localization is required for activity. For example, the CRN8 effector from P. infestans as well as other related CRN effectors specifically localize to plant nuclei (Stam et al.
2013; Van Damme et al. 2012). For CRN8, it was shown that its nuclear localization was required for the induction of plant cell death (Van Damme et al. 2012). Other effectors, such as Avh241 from P. sojae (Yu et al. 2012) and HaRxL17 from Hyaloperonospora arabidopsidis (Caillaud et al. 2012), are membrane associated. Deletion of an N-terminal region of Avh241 abolished both cell death activation as well as plasma membrane localization, indicating that effector activity is linked to subcellular localization. It is likely that subcellular localization of effectors reflects the interaction with host targets or host helper proteins in or at specific cellular compartments. The HopQ1 effector from Pseudomonas syringae pv. phaseolicola localizes to the host cytoplasm where it interacts with 143-3 plant proteins (Giska et al. 2013). The HopQ1-S51A mutant does not interact with the 14-3-3 proteins and accumulates in the plant nucleus rather than in the cytoplasm. Thus, it is possible that the binding to $14-3-3$ proteins affects the localization of the effector. Therefore, the distinct subcellular localization patterns of Mp10 and Mp42 suggest that these proteins affect aphid performance through different intracellular activities, probably toward distinct cellular targets. Mp42 is associated with plasma membranes and forms aggregates that colocalize with the ER, which may reflect disruption of the host membranes by this candidate effector. This disruption of host organelles by Mp42 may be responsible for the observed reduction in aphid fecundity upon in planta overexpression. On the other hand, this disruption by Mp42 may reflect a virulence activity of Mp42 important during aphid infestation, when Mp42 is present at much lower levels, or when other effectors could suppress any potential recognition of Mp42 as further detailed below.

Mp10 but not Mp42 triggered defense marker genes in the JA- and SA-signaling pathways as well as accumulation of the PR-1 protein. For several of the marker genes, the expression levels were somewhat reduced upon overexpression of Mp42 at later time points. It is possible that the observed disruption to host cell membranes by Mp42 is responsible for this observation, but this remains to be investigated. Mp10 reduces aphid fecundity upon transient overexpression by Agrobacterium (Bos et al. 210), and our data here suggest that this activity may depend on the activation of different defense signaling pathways. This then raises the question of whether aphid infestation triggers or suppresses the same hormone signaling pathways and whether these pathways are involved in defense responses to aphids. A number of studies have previously reported on gene expression changes in Arabidopsis and potato upon interaction with $M$. persicae. In Arabidopsis, M. persicae feeding induced gene expression predominantly of markers of the SA-signaling pathway, including PR-1 (de Vos et al. 2005; Moran and Thompson 2001). Kerchev and associates (2013) did a detailed analyses of local versus systemic gene expression upon $M$. persicae feeding on Arabidopsis, which showed that, in local and systemic leaves SA-, ethylene (ET)-, and abscisic-acid-induced genes were expressed, while JA-responsive genes such as PDF1.2 and VSP2 were repressed. Also, in potato, the interaction with $M$. persicae triggered SA-responsive genes (Alvarez et al. 2012). In addition, a small number of ET-responsive genes and JA-responsive genes were upregulated. Although, in potato, the different hormone signaling pathways are to some extent activated during infestation, the SA-signaling pathway is the predominant one. The strong increase of the PR-1 transcript and protein levels in $N$. benthamiana upon overexpression of Mp10 is in line with this induction of the SA-signaling pathway during aphid attack. Also, our finding that Mp10 delays the infection process of Phytophthora capsici, a hemibiotrophic plant pathogen on many crops plants, including members of the family Solanaceae, and potentially affects virulence of Agrobacterium 
tumefaciens, suggests that this candidate effector strongly activates SA-dependent plant defense responses. Also, our data show that candidate effectors from a pest can activate defenses against a plant pathogen. Therefore, further in-depth candidate effector characterization studies promise to provide us with key insights into how it may be possible to generate effective resistance against multiple pests and pathogens.

It is possible that the induction of SA- and JA-signaling pathways by Mp10 is responsible for the negative effect of this candidate effector on aphid fecundity in overexpression assays. It has been speculated that the activation of SA-signaling pathways by aphids counters activation of JA-dependent defense responses that are effective against aphids (de Vos et al. 2007). However, a

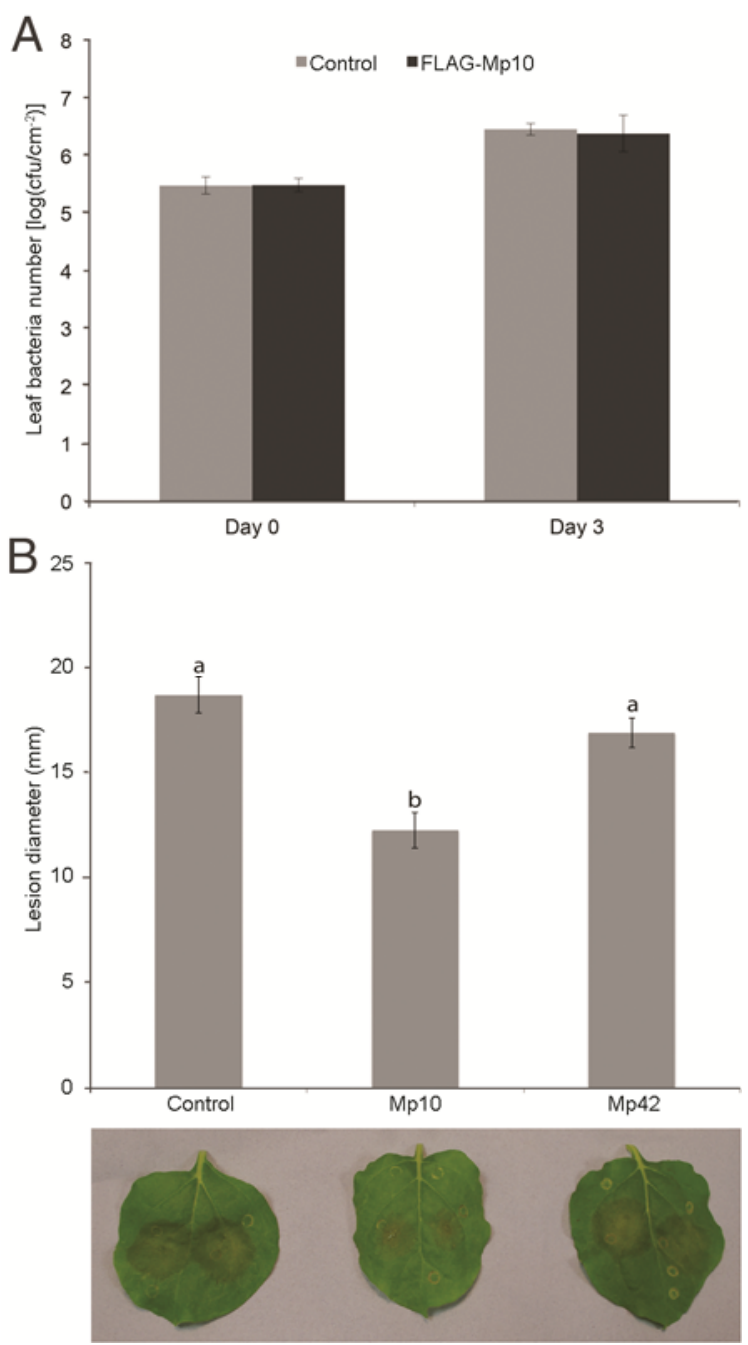

Fig. 5. Mp10 effects on Agrobacterium viability and host susceptibility to Phytophthora capsici. A, Agrobacterium growth in Nicotiana benthamiana leaves infiltrated with Agrobacterium strains carrying the vector pB7WGF2 together with strains expressing either FLAG-Mp10 or the vector control (control). Bacterial cell viability was monitored over 3 days by using antibiotics selecting for the $\mathrm{pB} 7 \mathrm{WGF} 2$ vector. Values represent the means of six samples from six different plants. Error bars indicate standard deviations. The experiment shown here is representative of three independent replicates. B, Lesion diameter after inoculation with $P$. capsici of plants overexpressing Mp10, Mp42, or the vector control. Lesion diameter was measured at 2 days after drop inoculation with $P$. capsici LT1534 spores. Graphs show the average lesion diameter from 10 infection sites. Error bars indicate standard deviations. One-way analysis of variance was performed to identify statistically significant differences. Bonferroni testing shows a significant difference $(P<0.01)$ between Mp10 and Mp42 and the corresponding vector control but not between Mp42 and the vector control. This result is representative of three independent replicates. recent study reported that Arabidopsis mutants with low ascorbate levels show increased SA signaling but also enhanced levels of resistance to $M$. persicae (Kerchev et al. 2013). This suggests that the activation of SA signaling does not necessarily suppress JA-dependent aphid defenses to promote virulence. The application of a benzothiadiazole (an SA analog) treatment in Arabidopsis plants resulted in a slight decrease in aphid reproduction (Moran and Thompson 2001). Also, the application of JA- and SA-inducting treatments in tomato resulted in increased levels of acquired resistance to aphids as well as $R$-gene (Mi-1.2)-mediated resistance, suggesting a role for both pathways in activation of defenses against aphids (Cooper et al. 2004). However, Arabidopsis mutants in the SA-signaling pathway, sid2 (SA-induction deficient 2) and nprl (nonexpressor of $P R-1$ ), as well as lines expressing the $N a h G$ gene, encoding a SA hydrolase, did not show altered susceptibility to $M$. persicae (Pegadaraju et al. 2005). On the other hand, Mewis and associates (2005), who used a different experimental set-up, observed a reduction in reproduction rates of $M$. persicae and Brevicoryne brassicae on the nprl mutant and on Arabidopsis plants carrying the $N a h G$ gene, suggesting that reduced SA levels corresponded with an increase in aphid resistance. Evidence for a role of JA in activation defenses against $M$. persicae in Arabidopsis was provided by Ellis and associates (2002), who showed that mutants with constitutive activation of JA signaling were more resistant to aphids than wild-type plants. It is likely that, in plant-aphid interactions, there is also a complex crosstalk and fine tuning between different signaling pathways, and pathways may function antagonistically or synergistically, depending on the timing, level, and sequence of responses (Pieterse et al. 2009). Furthermore, the role of signaling pathways may vary depending on the plant and aphid species interacting.

The induction of PAD4, a regulator of SA-signaling, by Mp10 could reflect the activation of defense responses active against $M$. persicae. Mutant pad4 Arabidopsis lines show increased levels of susceptibility to this aphid species (Pegadaraju et al. 2005), while overexpression of PAD4 in Arabidopsis results in increased levels of resistance (Pegadaraju et al. 2007). Interestingly, $P A D 4$-mediated resistance was determined to be phloem based and independent of EDS1 and SA signaling (Pegadaraju et al. 2005, 2007). Thus, it is possible that the induction of PAD4 by Mp10 affects aphid virulence on $N$. benthamiana in an SA-independent manner.

Both Mp10 and Mp42 seem to play a role in triggering different types of defense responses active against aphids, which raises the question of why they are expressed in the salivary glands and are potentially important to aphids. Although overexpression of these candidate effectors negatively impacts aphid fecundity, they may exhibit other activities that contribute to virulence during plant-aphid interactions. Indeed, Mp10 is known to suppress the ROS burst specifically triggered by the MAMP flg22 (Bos et al. 2010). However, it is possible that this activity is somehow linked to the observed activation of a range of plant defense responses by this candidate effector. Our data suggest that Mp10 impacts plant hormone signaling in transient overexpression assays, which potentially could affect other plant responses identified, such as PTI. Perception of flg22 involves the accumulation of SA (Tsuda et al. 2008); however, flg22-mediated PTI does not seem to strictly depend on the individual SA-, JA-, or ET-signaling pathways (Zipfel et al. 2004). Multiple-mutant analyses suggest that these pathways are involved in PTI to some extent (Tsuda et al. 2009). Although we cannot rule out that the suppression of the flg22triggered ROS by Mp10 results from its ability to activate various defense signaling pathways, it is possible that Mp10 has dual activities in activating and suppressing host defense responses. The strong activation of plant defenses by Mp10 
could reflect a recognition event by, for example, an NBSLRR protein, as suggested previously (Bos et al. 2010). In that case, the observed activation of plant defenses can be considered a genuine effector-mediated response. Perhaps other aphid effectors suppress this observed activation of defenses during compatible plant-aphid interactions so that Mp10 can contribute to virulence by suppressing PTI. It is also possible that the observed activation of defenses is an artifact of transient overexpression assays. For example, overexpression of an effector may result in excessive targeting of its virulence target or targeting of host proteins that are normally not targeted (Cunnac et al. 2009), leading to a misrepresentation of its actual virulence activity. Therefore, it is possible that the observed effects of Mp10 and Mp42 overexpression on activation or suppression of plant defenses, disruption of cell organelles, or aphid virulence are not representative of their true activities. Another explanation for the observed activation of defenses by Mp10 could be that this protein alters the basal immune response of the plant to Agrobacterium and, thereby, indirectly triggers defense-signaling pathways. However, we did not observe any negative effect of Mp10 on Agrobacterium cell viability. Moreover, JA signaling, although activated by $\mathrm{Mp} 10$ overexpression, does not play a major role in the Agrobacterium-Nicotiana interaction (Rico et al. 2010).

Research in the plant pathogen effector biology field, in many cases, involves transient assays based on Agrobacteriummediated expression, which provides an excellent tool to study effector activities but, as shown here, has its limitations. Our work shows that some (candidate) effectors such as Mp10 may directly affect their own expression mediated by Agrobacterium as well as the expression of other proteins. Therefore, care should be taken when performing assays to identify (candidate) effectors that suppress cell death triggered by elicitors, or (candidate) effectors that affect stability of a host protein. Without the appropriate controls included to test for specificity of the observed activity and for any effects the (candidate) effector may have on Agrobacterium-mediated expression, results can be misleading. With an increasing number of (candidate) effectors being characterized using these transient assays, it is essential to be aware that some of these proteins may directly affect methods used to investigate their activities. Despite this, the use of Agrobacterium-mediated expression here has allowed us to gain insight into the activities of two aphid effectors. Although these candidate effectors both showed the same phenotype in aphid fecundity assays they trigger different types of defense mechanisms. Future work will be aimed at identifying the potential host targets of these proteins to understand their role in modulating host cell processes.

\section{MATERIALS AND METHODS}

\section{Plasmid construction.}

The Mp10 and Mp42 genes (Supplementary Table S1) without signal peptide encoding sequences were amplified from $M$. persicae cDNA, cloned into the pENTR1a vector (Invitrogen, Carlsbad, CA, U.S.A.) using Gateway technology. BP recombination reactions were transformed in Escherichia coli XL1blue. Constructs were verified by sequencing. Subsequently, an LR recombination reaction was performed using $\mathrm{pB} 7 \mathrm{WG} 2$ (no tag), pB7WGF2 (N-terminal GFP tag) (Karimi et al. 2002), and pGWB12 (N-terminal FLAG tag) (Nakagawa et al. 2007) as destination vectors for expression in $N$. benthamiana.

\section{A. tumefaciens infiltration assays and plant growth.}

Constructs were introduced into A. tumefaciens strain GV3101 by electroporation. Transformants were selected using gentamycin $(12.5 \mu \mathrm{g} / \mathrm{ml})$, rifampicin $(50 \mu \mathrm{g} / \mathrm{ml})$, and spectino- mycin $(50 \mu \mathrm{g} / \mathrm{ml})$ for transformation into $\mathrm{pB} 7 \mathrm{WG} 2$ and $\mathrm{pB} 7 \mathrm{GWF} 2$, and gentamycin $(12.5 \mu \mathrm{g} / \mathrm{ml})$, rifampicin (50 $\mu \mathrm{g} / \mathrm{ml})$, and kanamycin $(50 \mu \mathrm{g} / \mathrm{ml})$ for transformation into pGWB12. For infiltration into leaves, recombinant strains of A. tumefaciens were grown in Luria-Bertani (LB) medium with above-mentioned antibiotics, harvested, and resuspended in Agrobacterium infiltration buffer (acetosyringone $125 \mu \mathrm{M}$ acetosyringone and $10 \mathrm{mM} \mathrm{MgCl}$ ) to reach a final concentration of an optical density at $600 \mathrm{~nm}\left(\mathrm{OD}_{600}\right)=0.3$ for Western blot analysis and $\mathrm{OD}_{600}=0.1$ for confocal imaging. Only for protein detection of the plant protein StCSN5 was the silencing suppressor p19 added to the Agrobacterium infiltration suspension. $N$. benthamiana plants were grown in a glasshouse for 4 to 6 weeks at 25 and $20^{\circ} \mathrm{C}$ for 16 and $8 \mathrm{~h}$, day and night, respectively. Infiltrations were performed using a syringe without a needle.

\section{Protein extractions and Western blots.}

Agroinfiltrated $N$. benthamiana leaves were harvested at 48, 72 , and $96 \mathrm{~h}$ postinfection (hpi) for time-course experiments or at 72 hpi for detection of protein expression. Proteins were extracted from leaf tissue with sodium dodecyl sulfate (SDS) sample buffer (with $50 \mathrm{mM}$ dithiothreitol) and boiled for $8 \mathrm{~min}$ before being loaded onto an SDS-polyacrylamide gel electrophoresis gel. After electrophoresis, proteins were transferred from the gel to a polyvinyl diflouride transfer membrane (Thermo Scientific, Waltham, MA, U.S.A.) using a transfer buffer (20 mM Tris and $150 \mathrm{mM}$ glycine). The membrane was later blocked using phosphate-buffered saline with Tween-20 $(0.1 \%)$ (PBS-T) plus $3 \%$ nonfat dry milk for $1 \mathrm{~h}$ at room temperature (RT) Immunoblot analysis was performed using antiGFP antibody (Santa Cruz, sc-8334; 1:5000) and anti-FLAG monoclonal antibodies (Santa Cruz, sc-166384; 1:5000) with incubation at $4^{\circ} \mathrm{C}$ overnight. The next day, the membrane was washed three times with PBS-T and incubated with goat antimouse (Santa Cruz, sc-2005) or goat anti-rabbit (Santa Cruz, sc-2004) at a concentration of $1: 10,000$ in PBS-T plus $3 \%$ nonfat milk at RT for $1 \mathrm{~h}$. The membrane was then washed four times (5 min each) with PBS-T and subjected to enhanced chemiluminscence reagent to detect the horseradish peroxidase signal. For enrichment using GFP-trap (Chromotek, PlaneggMartinsried, Germany), protein extraction was done with GTEN extraction buffer (10\% glycerol, $25 \mathrm{mM}$ Tris [pH 7.5], $1 \mathrm{mM}$ EDTA, and $150 \mathrm{mM} \mathrm{NaCl}$ ) and samples were incubated with GFP-trap beads at $4^{\circ} \mathrm{C}$ for $45 \mathrm{~min}$.

\section{Confocal microscopy.}

GFP (488-nm excitation; emission 500 to 300) and mRFP (561-nm excitation; emission 600 to 630) imaging in N. benthamiana was done with a confocal laser-scanning microscope (Leica TCS-SP2 AOBS) using HC PL FLUOTAR 63X0.9 and HCX APO L U-V 40X0.8 water-dipping lenses.

\section{Real-time PCR analyses.}

RNA was extracted from $N$. benthamiana leaves using the Spectrum Plant Total RNA kit (Sigma, St. Louis). Samples were quantified using a NanoDrop ND-1000 spectrophotometer (Thermo Scientific). To remove contaminating genomic DNA in RNA preparations, $1.5 \mu \mathrm{g}$ of total RNA was treated with $1 \mathrm{U}$ of RNase-free DNase I (Promega, Madison, WI, U.S.A.) according to the manufacturer's instructions. First-strand cDNA was synthesized using Moloney murine leukemia virus reverse transcriptase (Promega) and oligo (dT)18 primers (Invitrogen). Real-time PCR was performed in 25 - $\mu$ l reactions including cDNA, gene-specific primers, $12.5 \mu \mathrm{l}$ of SYBR Green mix (Qiagen, Hilden, Germany), and diethylpyrocarbonate-treated water (Invitrogen). PCR assays were done on a Chromo4 real- 
time detector (Bio-Rad, Hercules, CA, U.S.A.) under the following conditions: $95^{\circ} \mathrm{C}$ for $30 \mathrm{~s}, 40$ cycles at $95^{\circ} \mathrm{C}$ for $5 \mathrm{~s}$ and $60^{\circ} \mathrm{C}$ for $34 \mathrm{~s}$, followed by a dissociation step of $95^{\circ} \mathrm{C}$ for $15 \mathrm{~s}$, $60^{\circ} \mathrm{C}$ for $1 \mathrm{~min}$, and $95^{\circ} \mathrm{C}$ for $15 \mathrm{~s}$. Values are relative to the mean transcript abundance of actin.

\section{A. tumefaciens viability assays.}

Transformed A. tumefaciens strain GV3101 was grown at $28^{\circ} \mathrm{C}$ on $\mathrm{LB}$ medium containing the appropriate antibiotics for selection. An Agrobacterium strain carrying pB7WGF2 was coinfiltrated with strains carrying either pGWB12-Mp10 or pGWB12 (vector control). Infiltrations were done in $N$. benthamiana plants with a needleless syringe using $1 \times 10^{5} \mathrm{CFU} / \mathrm{ml}$ (per strain) bacterial suspensions in $10 \mathrm{mM} \mathrm{MgCl}_{2}$. Leaf tissue was harvested at 0,2 , and $4 \mathrm{dpi}$ and surface sterilized ( $1 \mathrm{~min}$ in $70 \%$ ethanol followed by $1 \mathrm{~min}$ in sterile distilled water). Then, leaf discs from each sample (with six replicates) were ground in $200 \mu \mathrm{l}$ of $10 \mathrm{mM} \mathrm{MgCl}_{2}$ using a tube pestle. Samples were vortex mixed, and 1:10 serial dilutions were performed and plated onto LB plates containing the appropriate antibiotics. Plates were incubated at $28^{\circ} \mathrm{C}$ for 2 days, at which time the CFU were counted. The experiment was repeated three times with similar results.

\section{P. capsici infection assays.}

Agroinfiltrated $N$. benthamiana leaves were detached after $48 \mathrm{~h}$ of infiltration. Leaves were placed on wet paper in a petri dish and drop inoculated with two $10-\mu$ d droplets of $P$. capsici LT1534 zoospores (500,000 spores/ml). The lesion diameter was measured at $48 \mathrm{hpi}$. One-way analysis of variance was performed to identify statistically significant differences.

\section{ACKNOWLEDGMENTS}

This work was supported the Biological Sciences Research Council (BBSRC grant BB/J005258/1) and the Royal Society of Edinburgh (cofunded by Marie Curie Actions). We thank E. Galiana for providing the PR-1 antibody; E. Gilroy and H. McLellan for their help with quantitative real-time PCR experiments; P. Boevink for providing the ER retention signal and, together with K. Wright, for advice and help with confocal imaging; E. Huitema for fruitful discussions and help with $P$. capsici assay; and P. Birch and J. Jones for useful comments and suggestions on this article. Conception and design or analysis and interpretation of data: P. A. Rodriguez, R. Stam, T. Warbroek, J. I. B. Bos; drafting the article or revising it critically for important intellectual content: P. A. Rodriguez, R Stam, J. I. B. Bos; final approval of the version to be published: P. A. Rodriguez, R. Stam, T. Warbroek, J. I. B. Bos.

\section{LITERATURE CITED}

Alvarez, A. E., Broglia, V. G., Alberti D’Amato, A. M., Wouters, D., van der Vossen, E., Garzo, E., Tjallingii, W. F., Dicke, M., and Vosman, B. 2012. Comparative analysis of Solanum stoloniferum responses to probing by the green peach aphid Myzus persicae and the potato aphid Macrosiphum euphorbiae. Insect Sci. 00:1-21.

Atamian, H. S., Chaudhary, R., Cin, V. D., Bao, E., Girke, T., and Kaloshian, I. 2013. In planta expression or delivery of potato aphid Macrosiphum euphorbiae effectors Me10 and Me23 enhances aphid fecundity. Mol. Plant-Microbe Interact. 26:67-74.

Blackman, R. L., and Eastop, V. F. 2006. Aphids on the World's Herbaceous Plants and Shrubs. John Wiley \& Sons, New York.

Bos, J. I. B., Prince, D., Pitino, M., Maffei, M. E., Win, J., and Hogenhout, S. A. 2010. A functional genomics approach identifies candidate effectors from the aphid species Myzus persicae (green peach aphid). PLoS Genet. 6:e1001216. Published online.

Caillaud, M. C., Piquerez, S. J. M., and Jones, J. D. G. 2012. Characterization of the membrane-associated HaRxL17 Hpa effector candidate. Plant Signal. Behav. 7:145-149.

Carolan, J. C., Fitzroy, C. I. J., Ashton, P. D., Douglas, A. E., and Wilkinson, T. L. 2009. The secreted salivary proteome of the pea aphid Acyrthosiphon pisum characterised by mass spectrometry. Proteomics 9:2457-2467.

Carolan, J. C., Caragea, D., Reardon, K. T., Mutti, N. S., Dittmer, N.,
Pappan, K., Cui, F., Castaneto, M., Poulain, J., Dossat, C., Tagu, D. Reese, J. C., Reeck, G. R., Wilkinson, T. L., and Edwards, O. R. 2011. Predicted effector molecules in the salivary secretome of the pea aphid (Acyrthosiphon pisum): a dual transcriptomic/proteomic approach. J. Proteome Res. 10:1505-1518.

Cooper, W. C., Jia, L., and Goggin, F. L. 2004. Acquired and $R$-genemediated resistance against the potato aphid in tomato. J. Chem. Ecol. 30:2527-2542.

Cunnac, S., Lindeberg, M., and Collmer, A. 2009. Pseudomonas syringae type III secretion system effectors: repertoires in search of functions. Curr. Opin. Microbiol. 12:53-60.

Denecke, J., De Rycke, R., and Botterman, J. 1992. Plant and mammalian sorting signals for protein retention in the endoplasmic reticulum contain a conserved epitope. EMBO (Eur. Mol. Biol. Organ.) J. 11:2345-2355.

de Vos, M., and Jander, G. 2009. Myzus persicae (green peach aphid) salivary components induce defence responses in Arabidopsis thaliana. Plant Cell Environ. 32:1548-1560.

de Vos, M., Van Oosten, V. R., Van Poecke, R. M., Van Pelt, J. A., Pozo, M. J., Mueller, M. J., Buchala, A. J., Métraux, J. P., Van Loon, L. C., Dicke, M., Pieterse, C. M. 2005. Signal signature and transcriptome changes of Arabidopsis during pathogen and insect attack. Mol. PlantMicrobe Interact. 18:923-937.

de Vos, M., Kim, J. H., and Jander, G. 2007. Biochemistry and molecular biology of Arabidopsis-aphid interactions. Bioessays 29:871-883.

Ellis, C., Karafyllidis, I., and Turner, J. G. 2002. Constitutive activation of jasmonate signaling in an Arabidopsis mutant correlates with enhanced resistance to Erysiphe cichoracearum, Pseudomonas syringae, and Myzus persicae. Mol. Plant-Microbe Interact. 15:1025-1030.

Giska, F., Lichocka, M., Piechocki, M., Dadlez, M., Schmelzer, E., Hennig, J., and Krzymowska, M. 2013. Phosphorylation of HopQ1, a type III effector from Pseudomonas syringae, creates a binding site for host 143-3 proteins. Plant Physiol. 161:2049-2061.

Hogenhout, S. A., and Bos, J. I. B. 2011. Effector proteins that modulate plant--insect interactions. Curr. Opin. Plant Biol. 14:422-428.

The International Aphid Genomics Consortium. 2010. Genome sequence of the pea aphid Acyrthosiphon pisum. PLoS Biol. 8:e1000313. Published online.

Jones, J. D. G., and Dangl, J. L. 2006. The plant immune system. Nature 444:323-329

Karimi, M., Inzé, D., and Depicker, A. 2002. GATEWAY vectors for Agrobacterium-mediated plant transformation. Trends Plant Sci. 7:193-195.

Kerchev, P. I., Karpinska, B., Morris, J. A., Hussain, A., Verrall, S. R., Hedley, P. E., Fenton, B., Foyer, C. H., and Hancock, R. D. 2013. Vitamin $\mathrm{C}$ and the abscisic acid-insensitive 4 (ABI4) transcription factor are important determinants of aphid resistance in Arabidopsis. Antiox. Redox Signal. 18:2091-2105.

Maimbo, M., Ohnishi, K., Hikichi, Y., Yoshioka, H., and Kiba, A. 2010. $\mathrm{S}$-glycoprotein-like protein regulates defense responses in Nicotiana plants against Ralstonia solanacearum. Plant Physiol. 152:2023-2035.

Mewis, I., Appel, H. M., Hom, A., Raina, R., and Schultz, J. C. 2005. Major signaling pathways modulate Arabidopsis glucosinolate accumulation and response to both phloem-feeding and chewing insects. Plant Physiol. 138:1149-1162.

Milling, A., Babujee, L., and Allen, C., 2011. Ralstonia solanacearum extracellular polysaccharide is a specific elicitor of defense responses in wilt-resistant tomato plants. PLoS One 6:e15853. Published online.

Moran, P. J., and Thompson, G. A. 2001. Molecular responses to aphid feeding in Arabidopsis in relation to plant defense pathways. Plant Physiol. 125:1074-1085.

Mutti, N. S., Louis, J., Pappan, L. K., Pappan, K., Begum, K., Chen, M. S., Park, Y., Dittmer, N., Marshall, J., Reese, J. C., and Reeck, G. R. 2008. A protein from the salivary glands of the pea aphid, Acyrthosiphon pisum, is essential in feeding on a host plant. Proc. Natl. Acad. Sci. U.SA. 105:9965-9969.

Nakagawa, T., Kurose, T., Hino, T., Tanaka, K., Kawamukai, M., Niwa, Y. Toyooka, K., Matsuoka, K., Jinbo, T., and Kimura, T. 2007. Development of series of gateway binary vectors, pGWBs, for realizing efficient construction of fusion genes for plant transformation. J. Biosci. Bioeng. 104:34-41.

Nicholson, S. J., Hartson, S. D., and Puterka, G. J. 2012. Proteomic analysis of secreted saliva from Russian Wheat Aphid (Diuraphis noxia Kurd.) biotypes that differ in virulence to wheat. J. Proteom. 75:22522268

Pegadaraju, V., Knepper, C., Reese, J., and Shah, J. 2005. Premature leaf senescence modulated by the Arabidopsis PHYTOALEXIN DEFICIENT4 gene is associated with defense against the phloem-feeding green peach aphid. Plant Physiol. 139:1927-1934.

Pegadaraju, V., Louis, J., Singh, V., Reese, J. C., Bautor, J., Feys, B. J., Cook, G., Parker, J. E., and Shah, J. 2007. Phloem-based resistance to green peach aphid is controlled by Arabidopsis PHYTOALEXIN DEFI- 
CIENT4 without its signaling partner ENHANCED DISEASE SUSCEPTIBILITY1. Plant J. 52:332-341.

Pieterse, C. M. J., Leon-Reyes, A., Van der Ent, S., and Van Wees, S. C. M. 2009. Networking by small-molecule hormones in plant immunity. Nat. Chem. Biol. 5:308-316.

Pitino, M., and Hogenhout, S. A. 2013. Aphid protein effectors promote aphid colonization in a plant species-specific manner. Mol. PlantMicrobe Interact. 26:130-139.

Ramsey, J. S., Wilson, A. C. C., de Vos, M., Sun, Q., Tamborindeguy, C., Winfield, A., Malloch, G., Smith, D. M., Fenton, B., Gray, S. M., and Jander, G. 2007. Genomic resources for Myzus persicae: EST sequencing, SNP identification, and microarray design. BMC Genomics 8:423.

Rico, A., Bennett, M. H., Forcat, S., Huang, W. E., and Preston, G. M. 2010. Agroinfiltration reduces ABA levels and suppresses Pseudomonas syringae-elicited salicylic acid production in Nicotiana tabacum. PLoS One 5:e8977. Published online.

Rodriguez, P. A., and Bos, J. I. B. 2013. Toward understanding the role of aphid effectors in plant infestation. Mol. Plant-Microbe Interact. 26:25-30.

Stam, R., Jupe, J., Howden, A. J. M., Morris, J. A., Boevink, P. C., Hedley, P. E., and Huitema, E. 2013. Identification and characterisation of CRN effectors in Phytophthora capsici shows modularity and functional diversity. PLoS One 8:e59517. Published online.

Tjallingii, W. F. 2006. Salivary secretions by aphids interacting with proteins of phloem wound responses. J. Exp. Bot. 57:739-745.

Tsuda, K., Sato, M., Glazebrook, J., Cohen, J. D., and Katagiri, F. 2008.
Interplay between MAMP-triggered and SA-mediated defense responses. Plant J. 53:763-775.

Tsuda, K., Sato, M., Stoddard, T., Glazebrook, J., and Katagiri, F. 2009. Network properties of robust immunity in plants. PLoS Genet. 5:e1000772. Published online.

Van Damme, M., Bozkurt, T. O., Cakir, C., Schornack, S., Sklenar, J. Jones, A. M. E., and Kamoun, S. 2012. The Irish potato famine pathogen Phytophthora infestans translocates the CRN8 kinase into host plant cells. PLoS Pathog. 8:e1002875. Published online.

Vida, T. A., and Emr, S. D. 1995. A new vital stain for visualizing vacuolar membrane dynamics and endocytosis in yeast. J. Cell Biol. 128:779792.

Will, T., Tjallingii, W. F., Thönnessen, A., and Van Bel, A. J. E. 2007. Molecular sabotage of plant defense by aphid saliva. Proc. Natl. Acad. Sci. U.SA. 104:10536-10541.

Yoon, J., Chung, W. I., and Doi, D. 2009. NbHB1, Nicotiana benthamiana homeobox 1 , is a jasmonic acid-dependent positive regulator of pathogen-induced plant cell death. New Phytol. 184:71-84.

Yu, X., Tang, J., Wang, Q., Ye, W., Tao, K., Duan, S., Lu, C., Yang, X., Dong, S., Zheng, X., and Wang, Y. 2012. The RxLR effector Avh24 from Phytophthora sojae requires plasma membrane localization to induce plant cell death. New Phytol. 196:247-260.

Zipfel, C., Robatzek, S., Navarro, L., Oakeley, E. J., Jones, J. D. G., Felix, G., and Boller, T. 2004. Bacterial disease resistance in Arabidopsis through flagellin perception. Nature 428:764-767. 